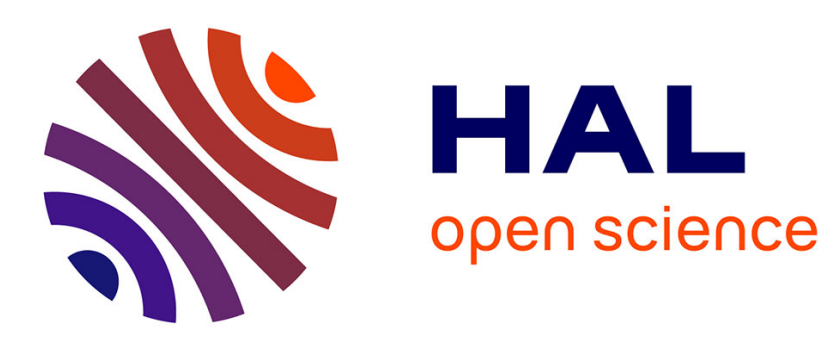

\title{
Bifurcation Equations through Multiple-Scales Analysis for a Continuous Model of a Planar Beam
}

\author{
Angelo Luongo, Angelo Di Egidio
}

\section{To cite this version:}

Angelo Luongo, Angelo Di Egidio. Bifurcation Equations through Multiple-Scales Analysis for a Continuous Model of a Planar Beam. Nonlinear Dynamics, 2005, 41 (1-3), pp.171-190. hal-00790794

\author{
HAL Id: hal-00790794 \\ https://hal.science/hal-00790794
}

Submitted on 21 Feb 2013

HAL is a multi-disciplinary open access archive for the deposit and dissemination of scientific research documents, whether they are published or not. The documents may come from teaching and research institutions in France or abroad, or from public or private research centers.
L'archive ouverte pluridisciplinaire HAL, est destinée au dépôt et à la diffusion de documents scientifiques de niveau recherche, publiés ou non, émanant des établissements d'enseignement et de recherche français ou étrangers, des laboratoires publics ou privés. 


\title{
Bifurcation Equations Through Multiple-Scales Analysis for a Continuous Model of a Planar Beam
}

\author{
ANGELO LUONGO and ANGELO DI EGIDIO* \\ Dipartimento di Ingegneria delle Strutture, delle Acque e del Terreno, Facoltà di Ingegneria - DISAT, p.le. E. Pontieri, \\ Università di L'Aquila, 670-40 Monteluco di Roio, L'Aquila, Italy; \\ "Author for correspondence (e-mail: ade @ing.univaq.it; fax: +39-0862-434548)
}

\begin{abstract}
The Multiple-Scale Method is applied directly to a one-dimensional continuous model to derive the equations goveming the asymptotic dynamic of the system around a bifurcation point. The theory is illustrated with reference to a specific example, namely an intemally constrained planar beam, equipped with a lumped viscoelastic device and loaded by a follower force. Nonlinear, integro-differential equations of motion are derived and expanded up to cubic terms in the transversal displacements and velocities of the beam. They are put in an operator form incorporating the mechanical boundary conditions, which account for the lumped viscoelastic device; the problem is thus governed by mixed algebraic-integro-differential operators. The linear stability of the trivial equilibrium is first studied. It reveals the existence of divergence, Hopf and double-zero bifurcations. The spectral properties of the linear operator and its adjoint are studied at the bifurcation points by obtaining closed-form expressions. Notably, the system is defective at the double-zero point, thus entailing the need to find a generalized eigenvector. A multiple-scale analysis is then performed for the three bifurcations and the relevant bifurcation equations are derived directly in their normal forms. Preliminary numerical results are illustrated for the double-zero bifurcation.
\end{abstract}

Key words: bifurcation, continuous model, divergence, double-zero bifurcation, Hopf, Multiple-Scale Method

\section{Introduction}

Reduction methods play a fundamental role in nonlinear dynamics. They allow the essential dynamics of the original system to be captured using models with a very low number of degrees of freedom, thus avoiding the use of brute numerical modeling.

Reduction methods have been thoroughly discussed by Steindl and Troger [1], who compared linear and nonlinear Galerkin methods, the center manifold and the approximate inertial manifold theories. Nayfeh [2] also developed a modified version of the Galerkin method, able to overcome the shortcomings of the classical procedure. Nayfeh and co-workers [3-6] have also widely applied the Multiple-Scale Method in the so called direct form, i.e. by dealing with the partial differential equations and boundary conditions rather than with algebraic systems obtained by means of a priori discretization. Within the framework of bifurcation theory, the writers have systematically applied the Multiple-Scale Method to finite-dimensional systems to derive the relevant bifurcation equations [7-12]. The method makes it possible to avoid both the search for the center manifold and the use of the normal form theory, since the algorithm furnishes bifurcation equations directly in normal form.

In this paper an attempt is made to extend the method to infinite-dimensional systems. However, some algorithmic questions arise in passing from discrete to continuous systems. The first concerns the way to enforce solvability to the singular non-homogeneous boundary value problems appearing at the different orders of the perturbation procedures. According to functional analysis, a suitable change of variables should be performed to render homogeneous the boundary conditions, and then orthogonality 
required between the known term of the field equation and the solutions of the homogeneous adjoint problem. A more effective way to derive the solvability conditions is applied by Nayfeh [13], who uses the bilinear identity accounting for all non-homogeneous terms, both in the field and on the boundary. However, since the conditions consist in a volume integral plus a surface integral, they are not in the scalar product form from which the adjoint problem was derived. To overcome this formal problem and to preserve a strict analogy with algebraic problems, a different approach will be followed here. Namely, the boundary conditions will be appended at the field equations by incorporating them in an augmented operator acting on the field variable as well as on its restriction at the boundary, introduced as further dummy variable. Accordingly, the scalar product incorporates the surface integral.

A second problem consists in the possibility to extend the properties, well known in algebra, of the Jordan chain of generalized eigenvectors to differential operators. These eigenvectors emerge naturally when the method is applied to defective bifurcations, i.e. bifurcations occurring for multiple eigenvalues and linear non-self-adjoint operators (see $[10,11]$ in which a discrete system is considered). In an initial attempt to solve the problem, the writers assumed that the chain also exists for continuous systems. The assumption was grounded on an engineering approach, according to which a continuous system can be modeled by an arbitrarily large discrete system, for which such chains do exist. This assumption was recently supported by two papers by Kirillov and Seyranian [14, 15], which cite a paper by Keldysh [16] (written in Russian and unknown to the authors of the present paper), who first extended the concept of Jordan chains to a wide class of non-self-adjoint operators. In [14] Keldysh chains are used to solve a linear differential problem in the same way that Jordan chains are used in linear algebraic problems. The same is made in this paper.

The paper is organized as follows. In Section 2 a nonlinear structural model is described and the equations of motion derived. In Section 3 the linear problem is dealt with to analyze stability of the trivial equilibrium; moreover, the adjoint problem is derived. Section 4 is devoted to bifurcation analysis, performed by the Multiple-Scale Method, for both codimension-1 (divergence and Hopf) and codimension-2 (double-zero) bifurcations, exhibited by the system. Preliminary numerical results are displayed in Section 5 for the multiple bifurcation. Finally, some conclusions are drawn in Section 6, where the algorithm is summarized.

\section{Model}

A planar, inextensible and shear-undeformable straight beam is considered, fixed at end $A$, constrained by a linear viscoelastic device at end $B$ and loaded by a follower force $P$ at $B$ (Figure 1). The device consists of an extensional spring of stiffness $k_{\mathrm{e}}$ and two dashpots, of constants $c_{\mathrm{e}}$ and $c_{\mathrm{t}}$ of an extensional and a torsional type, respectively.

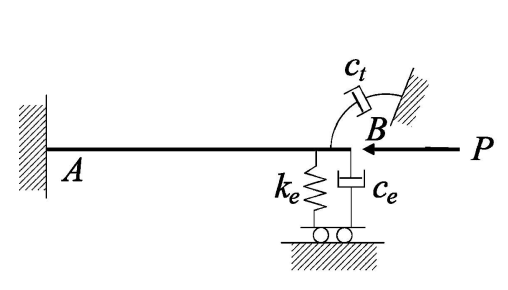

(a)

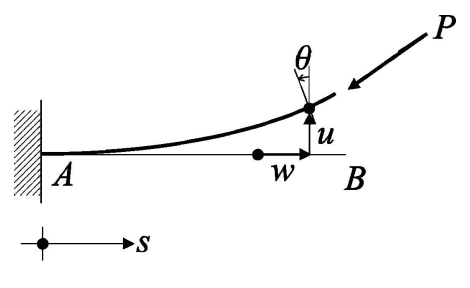

(b)

Figure 1. Model and displacements. 
The actual configuration of the beam is described by the transversal displacement field $u(s)$, the longitudinal displacement $w(s)$ and the rotation $\vartheta(s)$, where $s \in[0, l]$ is an abscissa. The three displacements, however, are not independent because of the internal constraints:

$$
\sin \vartheta=u^{\prime} ; \quad \varepsilon:=\sqrt{\left(1+w^{\prime}\right)^{2}+u^{\prime 2}}-1=0
$$

expressing shear-undeformability and inextensibility, respectively. The curvature $\kappa(s)$ is assumed as the (unique) strain measure; from Equation $\left(1_{1}\right)$ it follows that

$$
\kappa:=\vartheta^{\prime}=\frac{u^{\prime \prime}}{\sqrt{1-u^{\prime 2}}}
$$

The equations of motion are derived by the generalized Hamiltonian principle, introducing the constraint equation $\left(1_{2}\right)$ by a Lagrangian multiplier $\lambda(s)$, having the meaning of axial (reactive) force. The variational principle reads:

$$
\begin{aligned}
\delta H= & \int_{t_{1}}^{t_{2}} \int_{0}^{l}[m(\dot{u} \delta \dot{u}+\dot{w} \delta \dot{w})-E I \kappa \delta \kappa-\delta(\lambda \varepsilon)] d s d t \\
& -\int_{t_{1}}^{t_{2}}\left[\left(k_{\mathrm{e}} u_{B}\right) \delta u_{B}+\left(c_{\mathrm{e}} \dot{u}_{B}\right) \delta u_{B}+\left(c_{\mathrm{t}} \dot{\vartheta}_{B}\right) \delta \vartheta_{B}\right] d t \\
& +\int_{t_{1}}^{t_{2}}\left(P \cos \vartheta_{B}\right) \delta w_{B}+\left(P \sin \vartheta_{B}\right) \delta u_{B}=0 \quad \forall(\delta u, \delta w, \delta \vartheta, \delta \lambda)
\end{aligned}
$$

where the index $B$ denotes evaluation at $s=l$. By using Equation $\left(1_{1}\right)$ to eliminate the rotation $\vartheta(s)$ and expanding $u(s)$ in Taylor series, the equations of motion, corrected up to the third-order, are derived. By introducing the following non-dimensional quantities:

$$
\begin{array}{rlrl}
\tau & =\omega t ; \quad \tilde{s}=s / l ; \quad \tilde{u}=u / l ; & \tilde{w}=w / l ; \\
\omega^{2}=E I / m l^{4} ; \quad 2 \mu=P l^{2} / E I ; & \tilde{\lambda}=\lambda / m l^{2} \omega^{2} ; \\
\kappa & =k_{\mathrm{e}} l^{3} / E I ; \quad \xi_{\mathrm{e}}=c_{\mathrm{e}} \omega l^{3} / E I ; & \xi_{\mathrm{t}}=c_{\mathrm{t}} \omega l / E I
\end{array}
$$

and omitting the tilde symbol, they read:

$$
\begin{aligned}
\ddot{w}-\lambda^{\prime} & =0 \\
\ddot{u}+u^{\mathrm{IV}}+\left[u^{\prime}\left(u^{\prime} u^{\prime \prime}\right)^{\prime}\right]^{\prime}-\left(\lambda u^{\prime}\right)^{\prime} & =0 \\
w^{\prime}+u^{\prime 2} / 2 & =0
\end{aligned}
$$

with the relevant boundary conditions of a geometrical type:

$$
w_{A}=0, \quad u_{A}=0, \quad u_{A}^{\prime}+1 / 6 u_{A}^{\prime 3}=0
$$

and a mechanical type:

$$
\begin{array}{r}
\lambda_{B}+\mu\left(2-u_{B}^{\prime 2}\right)=0, \quad\left(u_{B}^{\prime \prime}+u_{B}^{\prime \prime} u_{B}^{\prime 2}\right)+\xi_{t}\left(\dot{u}_{B}^{\prime}+2 u_{B}^{\prime 2} \dot{u}_{B}^{\prime}\right)=0 \\
\left(u_{B}^{\prime \prime \prime}+u_{B}^{\prime \prime \prime} u_{B}^{\prime 2}+u_{B}^{\prime \prime 2} u_{B}^{\prime}\right)-\kappa u_{B}-\xi_{\mathrm{e}} \dot{u}_{B}-\lambda_{B} u_{B}^{\prime}-2 \mu u_{B}^{\prime}=0
\end{array}
$$


The longitudinal displacement $w(s)$ and the axial force $\lambda(s)$ are then condensed by integrating Equation $\left(5_{3}\right)$ and $\left(5_{1}\right)$ with the boundary conditions $\left(6_{1}\right)$ and $\left(7_{1}\right)$ :

$$
w=-\int_{0}^{s} \frac{u^{\prime 2}}{2} d s ; \quad \lambda=\int_{1}^{s}\left(-\int_{0}^{s} \frac{u^{\prime 2}}{2} d s\right) d s-\mu\left(2-u_{B}^{\prime 2}\right)
$$

By substituting Equation $\left(8_{2}\right)$ in Equation $\left(5_{2}\right)$ and the remaining boundary conditions, the following condensed equations in the unique variable $u(s)$ are finally derived:

$$
\begin{array}{r}
\ddot{u}+u^{\mathrm{IV}}+\left[u^{\prime}\left(u^{\prime} u^{\prime \prime}\right)^{\prime}\right]^{\prime}+2 \mu\left(1-u_{B}^{\prime 2} / 2\right) u^{\prime \prime}-\left[\left(\int_{1}^{s}\left(\int_{0}^{s} u^{\prime 2} / 2 d s\right) d s\right) u^{\prime}\right]^{\prime}=0 \\
u_{A}=0, \quad u_{A}^{\prime}=0 \\
\left(u_{B}^{\prime \prime}+u_{B}^{\prime \prime} u_{B}^{\prime 2}\right)+\xi_{\mathrm{t}}\left(\dot{u}_{B}^{\prime}+2 u_{B}^{\prime 2} \dot{u}_{B}^{\prime}\right)=0, \\
\left(u_{B}^{\prime \prime \prime}+u_{B}^{\prime \prime \prime} u_{B}^{\prime 2}+u_{B}^{\prime \prime 2} u_{B}^{\prime}\right)-\kappa u_{B}-\xi_{\mathrm{e}} \dot{u}_{B}-2 \mu\left(u_{B}^{\prime 2} / 2\right) u_{B}^{\prime}=0
\end{array}
$$

They are of an integro-differential type and contain only cubic nonlinearities.

For further developments, the previous equations are more conveniently recast in the following operator form, in which the mechanical conditions are incorporated in the operators:

$$
\begin{aligned}
\mathbf{M u}+\mathbf{C u}+\mathbf{K u} & =\mathbf{n}\left((\mathbf{u}, \dot{\mathbf{u}})^{3}\right) \\
\mathbf{g u} & =\mathbf{0}
\end{aligned}
$$

where

$$
\begin{gathered}
\mathbf{u}=\left\{\begin{array}{c}
u \\
u_{B} \\
u_{B}^{\prime}
\end{array}\right\} ; \mathbf{M}=\left[\begin{array}{lll}
1 & 0 & 0 \\
0 & 0 & 0 \\
0 & 0 & 0
\end{array}\right] ; \mathbf{C}=\left[\begin{array}{ccc}
0 & 0 & 0 \\
0 & \xi_{\mathrm{e}} & 0 \\
0 & 0 & \xi_{\mathrm{t}}
\end{array}\right] ; \mathbf{K}=\left[\begin{array}{ccc}
D^{4}+2 \mu D^{2} & 0 & 0 \\
-D_{B}^{3} & \kappa & 0 \\
D_{B}^{2} & 0 & 0
\end{array}\right] ; \mathbf{g u}=\left\{\begin{array}{l}
u_{A} \\
u_{A}^{\prime}
\end{array}\right\} \\
\mathbf{n}\left((\mathbf{u}, \dot{\mathbf{u}})^{3}\right)=\left\{\begin{array}{c}
-\left[u^{\prime}\left(u^{\prime} u^{\prime \prime}\right)^{\prime}\right]^{\prime}+\mu u_{B}^{\prime 2} u^{\prime \prime}-\left[\int_{1}^{s}\left(\int_{0}^{s} \frac{1}{2} u^{\prime 2} d s\right)^{*} d s\right] u^{\prime \prime}-\left[\left(\int_{0}^{s} \frac{1}{2} u^{\prime 2} d s\right)^{*}\right] u^{\prime} \\
+u_{B}^{\prime \prime \prime} u_{B}^{\prime 2}+u^{\prime \prime 2}{ }_{B}^{\prime} u_{B}^{\prime}-\mu u_{B}^{\prime 2} u_{B}^{\prime} \\
-u_{B}^{\prime \prime} u_{B}^{\prime 2}-\xi_{\mathrm{t}} 2 u_{B}^{\prime 2} \dot{u}_{B}^{\prime}
\end{array}\right\}
\end{gathered}
$$

with $D^{k}=d^{k} / d s^{k}$, $\mathbf{g}$ being the geometrical operator at the boundary $s=0$ and $\mathbf{n}\left((\mathbf{u}, \dot{\mathbf{u}})^{3}\right)$ a homogeneous

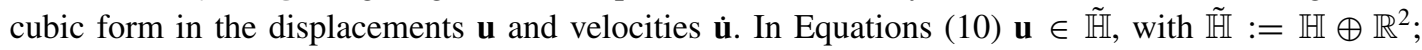
consequently, the mass, damping and stiffness operators $\mathbf{M}, \mathbf{C}$ and $\mathbf{K}$ are mixed differential-algebraic operators. Equations (10) can also be rewritten in a first-order form by taking displacements $\mathbf{u}$ and velocities $\hat{\mathbf{u}}:=\dot{\mathbf{u}}$ as state variables. They read:

$$
\begin{aligned}
& \mathbf{B} \dot{\mathbf{U}}=\mathbf{A} \mathbf{U}+\mathbf{N}\left(\mathbf{U}^{3}\right) \\
& \mathbf{G U}=\mathbf{0}
\end{aligned}
$$




$$
\mathbf{U}=\left\{\begin{array}{l}
\mathbf{u} \\
\hat{\mathbf{u}}
\end{array}\right\} ; \hat{\mathbf{u}}=\left\{\begin{array}{c}
\dot{u} \\
\dot{u}_{B} \\
\dot{u}_{B}^{\prime}
\end{array}\right\} ; \mathbf{B}=\left[\begin{array}{cc}
\mathbf{I} & \mathbf{0} \\
\mathbf{0} & \mathbf{M}
\end{array}\right] ; \mathbf{A}=\left[\begin{array}{cc}
\mathbf{0} & \mathbf{I} \\
-\mathbf{K} & -\mathbf{C}
\end{array}\right] ; \mathbf{N}\left(\mathbf{U}^{3}\right)=\left\{\begin{array}{c}
\mathbf{0} \\
\mathbf{n}\left(\mathbf{U}^{3}\right)
\end{array}\right\} ; \mathbf{G U}=\left\{\begin{array}{l}
u_{A} \\
u_{A}^{\prime}
\end{array}\right\}
$$

with $\mathbf{U} \in \tilde{\mathbb{H}}^{2}$ being the state-variable vector.

\section{Linear Stability Problem}

\subsection{Right AND LEFT EIgENVALUE ProblemS}

The linearized equations of motion admit the solution:

$$
\mathbf{U}=\boldsymbol{\Phi} e^{\lambda t}
$$

where $\Phi$ and $\lambda$ satisfy the following (right) eigenvalue problem:

$$
\begin{aligned}
(\mathbf{A}-\lambda \mathbf{B}) \boldsymbol{\Phi} & =\mathbf{0} \\
\mathbf{G} \boldsymbol{\Phi} & =\mathbf{0}
\end{aligned}
$$

By making the partition $\boldsymbol{\Phi}=\{\boldsymbol{\varphi} \hat{\varphi}\}^{\mathrm{T}}$, with $\varphi=\left\{\varphi \varphi_{B} \varphi_{B}^{\prime}\right\}^{\mathrm{T}} \in \tilde{\mathbb{H}}$ and $\hat{\varphi}=\left\{\hat{\varphi} \hat{\varphi}_{B} \hat{\varphi}_{B}^{\prime}\right\}^{\mathrm{T}} \in \tilde{\mathbb{H}}$ and accounting for Equations (13), Equations (15) read:

$$
\begin{aligned}
\hat{\varphi} & =\lambda \boldsymbol{\varphi} \\
\mathbf{K} \varphi+\mathbf{C} \hat{\varphi}+\lambda \mathbf{M} \hat{\varphi} & =\mathbf{0} \\
\varphi_{A}=0 ; \quad \varphi_{A}^{\prime} & =0
\end{aligned}
$$

An inner product is then introduced, in $\tilde{\mathbb{H}}$ or $\tilde{\mathbb{H}}^{2}$, as:

$$
\begin{aligned}
(\boldsymbol{\varphi}, \boldsymbol{\psi}) & :=\int_{0}^{1} \bar{\varphi}_{1}(s) \psi_{1}(s) d s+\sum_{j=2,3} \bar{\varphi}_{j} \psi_{j} \quad \boldsymbol{\varphi}, \boldsymbol{\psi} \in \tilde{\mathbb{H}} \\
(\boldsymbol{\Phi}, \boldsymbol{\Psi}) & :=\int_{0}^{1} \sum_{i=1,4} \overline{\boldsymbol{\Phi}}_{i}(s) \Psi_{i}(s) d s+\sum_{j=2,3,5,6} \bar{\Phi}_{j} \Psi_{j} \quad \boldsymbol{\Phi}, \boldsymbol{\Psi} \in \tilde{\mathbb{H}}^{2}
\end{aligned}
$$

and from the bilinear identity,

$$
(\Psi,(\mathbf{A}-\lambda \mathbf{B}) \Phi)=\left(\left(\mathbf{A}^{*}-\bar{\lambda} \mathbf{B}^{*}\right) \Psi, \Phi\right)
$$

the adjoint operators $\mathbf{A}^{*}, \mathbf{B}^{*}$ and adjoint boundary conditions $\mathbf{G}^{*} \boldsymbol{\Psi}$ are derived:

$$
\mathbf{B}^{*}=\left[\begin{array}{cc}
\mathbf{I} & \mathbf{0} \\
\mathbf{0} & \mathbf{M}
\end{array}\right] ; \mathbf{A}^{*}=\left[\begin{array}{cc}
\mathbf{0} & -\mathbf{K}^{*} \\
\mathbf{I} & -\mathbf{C}^{*}
\end{array}\right] ; \mathbf{G}^{*} \boldsymbol{\Psi}=\left\{\begin{array}{c}
\psi_{A} \\
-\psi_{A}^{\prime}
\end{array}\right\}
$$




$$
\mathbf{C}^{*}=\mathbf{C}=\left[\begin{array}{lll}
0 & 0 & 0 \\
0 & \xi_{\mathrm{e}} & 0 \\
0 & 0 & \xi_{\mathrm{t}}
\end{array}\right] ; \quad \mathbf{K}^{*}=\left[\begin{array}{ccc}
D^{4}+2 \mu D^{2} & 0 & 0 \\
-D_{B}^{3} & \kappa & -2 \mu \\
D_{B}^{2} & 2 \mu & 0
\end{array}\right]
$$

and $\boldsymbol{\Psi}=\{\hat{\boldsymbol{\psi}} \boldsymbol{\psi}\}^{\mathrm{T}}{ }^{1}$. It turns out that while $\mathbf{C}$ is self-adjoint, $\mathbf{K}$ is not self-adjoint because of the presence of the follower force $\mu$. The adjoint (or left) eigenvalue problem therefore reads:

$$
\begin{aligned}
\left(\mathbf{A}^{*}-\bar{\lambda} \mathbf{B}^{*}\right) \boldsymbol{\Psi} & =\mathbf{0} \\
\mathbf{G}^{*} \boldsymbol{\Psi} & =\mathbf{0}
\end{aligned}
$$

or

$$
\begin{aligned}
& \mathbf{K}^{*} \boldsymbol{\psi}+\bar{\lambda} \hat{\boldsymbol{\psi}}=\mathbf{0} \\
& \hat{\boldsymbol{\psi}}-\mathbf{C}^{*} \boldsymbol{\psi}-\bar{\lambda} \mathbf{M} \boldsymbol{\psi}=\mathbf{0} \\
& \psi_{A}=0 ; \quad \psi_{A}^{\prime}=0
\end{aligned}
$$

In the following, two critical cases are considered:

(a) $\lambda$ is a simple eigenvalue, namely $\lambda=0$ (divergence bifurcation) or $\lambda= \pm \mathrm{i} \omega$ (simple Hopf bifurcation);

(b) $\lambda$ is a double-zero eigenvalue (Takens-Bogdanova bifurcation).

In case (a), a right eigenvector $\boldsymbol{\Phi}=\{\boldsymbol{\varphi} \lambda \varphi\}^{\mathrm{T}}$ and a left eigenvector $\boldsymbol{\Psi}=\left\{\left(\mathbf{C}^{*}+\bar{\lambda} \mathbf{M}\right) \boldsymbol{\psi} \boldsymbol{\psi}\right\}^{\mathrm{T}}$ are associated with $\lambda$, with $(\Psi, \Phi) \neq 0$. They can be normalized by requiring $\varphi_{B}=1$ and $(\boldsymbol{\Psi}, \boldsymbol{\Phi})=1$. In case (b), in contrast, just one (proper) right eigenvector $\Phi_{1}=\left\{\varphi_{1} \mathbf{0}\right\}^{\mathrm{T}}$ and one (proper) left eigenvector $\boldsymbol{\Psi}_{2}=\left\{\mathbf{C}^{*} \boldsymbol{\psi}_{2} \boldsymbol{\psi}_{2}\right\}^{\mathrm{T}}$ are found, with $\left(\boldsymbol{\Psi}_{2}, \boldsymbol{\Phi}_{1}\right)=\left(\mathbf{C}^{*} \boldsymbol{\psi}_{2}, \boldsymbol{\varphi}_{1}\right)=0$. To complete the base, a generalized, index-two, right eigenvector $\Phi_{2}=\left\{\varphi_{2} \hat{\varphi}_{2}\right\}^{\mathrm{T}}$ must be evaluated by the following non-homogeneous problem:

$$
\begin{aligned}
& \mathbf{A} \boldsymbol{\Phi}_{2}=\boldsymbol{\Phi}_{1} \\
& \mathbf{G} \boldsymbol{\Phi}_{2}=\mathbf{0}
\end{aligned}
$$

equivalent to:

$$
\begin{aligned}
& \hat{\boldsymbol{\varphi}}_{2}=\boldsymbol{\varphi}_{1} \\
& -\mathbf{K} \boldsymbol{\varphi}_{2}-\mathbf{C} \hat{\boldsymbol{\varphi}}_{2}=\mathbf{0} \\
& \varphi_{2 A}=0 ; \quad \varphi_{2 A}^{\prime}=0
\end{aligned}
$$

The generalized eigenvector $\boldsymbol{\Phi}_{2}$ therefore consists of velocities $\hat{\varphi}_{2}$ equal to the static (buckling) displacements $\varphi_{1}$ and displacements $\varphi_{2}$ equal to the static response of the system to viscous forces $-\mathbf{C} \hat{\varphi}_{2} \equiv-\mathbf{C} \varphi_{1}$, triggered by buckling. The generalized eigenvector $\Phi_{2}$ is not unique, since the operator $\mathbf{A}$ is singular; to render it unique, a suitable normalization condition must be enforced, e.g. $\varphi_{2 B}=0$. After having normalized $\boldsymbol{\Phi}_{2}$, the left proper eigenvector $\boldsymbol{\Psi}_{2}$ is also normalized, by requiring $\left(\Psi_{2}, \Phi_{2}\right)=1$

\footnotetext{
${ }^{1}$ It should be noted that the order of the variables $\psi$ and $\hat{\psi}$ in $\Psi$ is exchanged with respect to $\varphi$ and $\hat{\varphi}$ in $\Phi$. Therefore, $(\Psi, \Phi)=(\hat{\psi}, \varphi)+(\psi, \hat{\varphi})$.
} 
The parameters $\mu$ and $\kappa$ are taken as bifurcation parameters and the boundaries at which the trivial equilibrium position loses stability are sought in the $(\kappa, \mu)$ parameter plane. The field equations in problem (16) admit the following solution, accounting for the geometrical boundary conditions $\left(16_{3,4}\right)$ at $A$ :

$$
\varphi(s)=c_{1}(\cos p s-\cosh q s)+c_{2}\left(\sin p s-\frac{p}{q} \sinh q s\right)
$$

where $c_{1}$ and $c_{2}$ are arbitrary constants and

$$
q^{2}:=\sqrt{\mu^{2}-\lambda^{2}}-\mu, \quad p^{2}:=\sqrt{\mu^{2}-\lambda^{2}}+\mu
$$

have been posed. With Equation (25), the two mechanical boundary conditions at $B$ read:

$$
\mathbf{R}(\lambda, \kappa, \mu) \mathbf{c}=\mathbf{0}
$$

where

$$
\begin{aligned}
\mathbf{R}(\lambda, \kappa, \mu) & =\left[\begin{array}{ll}
-\left(\kappa+\lambda \xi_{\mathrm{e}}\right)(\cos p-\cosh q) & -\left(\kappa+\lambda \xi_{\mathrm{e}}\right)(\sin p-p / q \sinh q) \\
+p^{3} \sin p-q^{3} \sinh q & -p^{3} \cos p-q^{2} p \cosh q \\
-\lambda \xi_{\mathrm{t}}(p \sin p+q \sinh q) & \lambda \xi_{\mathrm{t}} p(\cos p-\cosh q) \\
-p^{2} \cos p-q^{2} \cosh q & -p^{2} \sin p-p q \sinh q
\end{array}\right] \\
\mathbf{c} & =\left\{\begin{array}{l}
c_{1} \\
c_{2}
\end{array}\right\}
\end{aligned}
$$

By vanishing the determinant of $\mathbf{R}$, a transcendent equation in $\lambda$ follows depending on the bifurcation parameters; it is analyzed in the later section.

(a) Divergence boundary and double-zero point

If $\lambda \rightarrow 0$, then $p \rightarrow \sqrt{2 \mu}$ and $q \rightarrow \mathrm{i} \lambda / \sqrt{2 \mu}$. By substituting them in $\mathbf{R}$ and expanding its determinant in a Taylor series of $\lambda$, it follows:

$\operatorname{det} \mathbf{R}(\lambda, \kappa, \mu)=I_{0}+\lambda I_{1}+\lambda^{2} I_{2}+\cdots=0$

with $I_{j}=I_{j}(\kappa, \mu)$ as the invariant of the system. In particular, the leading invariants are found to be:

$$
\begin{aligned}
& I_{0}=2.82 \mu^{3 / 2} \kappa \cos (\sqrt{2 \mu})-5.66 \mu^{5 / 2}-2 \mu \kappa \sin (\sqrt{2 \mu}) \\
& I_{1}=2[\sqrt{2 \mu}(\mu \alpha+\kappa) \cos (\sqrt{2 \mu})-\kappa \sqrt{2 \mu}-\mu(2 \mu+\alpha-\kappa) \sin (\sqrt{2 \mu})]
\end{aligned}
$$

where $\alpha:=\xi_{\mathrm{e}} / \xi_{\mathrm{t}}$ has been posed. The divergence boundary $\mathcal{D}$ has codimension-1 and is defined by the equation $I_{0}(\kappa, \mu)=0$. The double-zero point has codimension- 2 and is determined by the simultaneous vanishing of the first two invariants, $I_{0}(\kappa, \mu)=0$ and $I_{1}(\kappa, \mu)=0$; therefore, it depends on the damping ratio $\alpha$ only and not on the values of the single coefficients $\xi_{\mathrm{e}}$ and $\xi_{\mathrm{t}}$. The divergence curve $\mathcal{D} \equiv \mathcal{I}_{0}$ and the double-zero point $\mathcal{I}_{0} \cap \mathcal{I}_{1}$ are displayed in Figure 2 for $\alpha=0.5$. The rightand left proper eigenvectors, both at a selected point $D \equiv(50,12.69)$ on the $\mathcal{D}$ curve and at the 


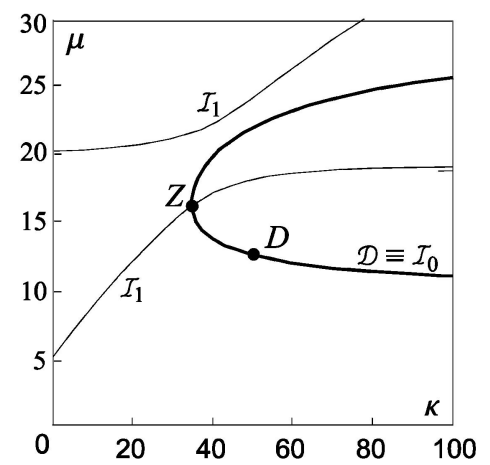

Figure 2. Evaluation of the double-zero point $Z$ : curves $I_{k}$ denote vanishing of the invariants $I_{k}$ of the characteristic equation; $\alpha=\xi_{\mathrm{e}} / \xi_{\mathrm{t}}=0.5$

double-zero point $Z \equiv(34.82,16.47)$ are reported in the appendix. There, the index-2 generalized eigenvector is also evaluated.

There are two additional methods for determining the double-zero point $Z$; they are based on one of the following properties holding at this point, namely:

(I) a generalized eigenvector $\boldsymbol{\Phi}_{2}$ exists (i.e. compatibility of Equations (24)) is satisfied;

(II) the proper eigenvectors are orthogonal (i.e. $\left(\boldsymbol{\Psi}_{2}, \boldsymbol{\Phi}_{1}\right)=0$ ).

Following method (I), when the field problem (24) is solved and the geometrical boundary conditions are accounted for, the mechanical conditions lead to the following algebraic non-homogeneous problem:

$$
\mathbf{R}_{0}(\kappa, \mu) \mathbf{a}=\mathbf{f}
$$

in which, by Equation (28), $\mathbf{R}_{0}:=\mathbf{R}(0, \kappa, \mu), \mathbf{a} \in \mathbb{R}^{2}$ are arbitrary constants and $\mathbf{f} \in \mathbb{R}^{2}$ are known terms. Since $\mathbf{R}_{0}$ is singular along the $\mathcal{D}$ curve, the problem (31) does not generally admit a solution (i.e. there is no generalized eigenvector $\boldsymbol{\Phi}_{2}$ at a generic divergence point). However, if the compatibility condition is also required:

$$
\mathbf{f}^{\mathrm{T}} \mathbf{a}^{*}=0 \quad \forall \mathbf{a}^{*}: \mathbf{R}_{0}^{\mathrm{T}} \mathbf{a}^{*}=\mathbf{0}
$$

the double-zero point $Z$ is recovered. There, Equation (31) can be solved and the generalized eigenvector $\boldsymbol{\Phi}_{2}$ evaluated to within one constant.

Following method (II), the condition $\left(\boldsymbol{\Psi}_{2}, \boldsymbol{\Phi}_{1}\right)=\left(\mathbf{C}^{*} \boldsymbol{\psi}_{2}, \boldsymbol{\varphi}_{1}\right)=0$ reads:

$$
\xi_{\mathrm{e}} \varphi_{1 B}+\psi_{2 B}+\xi_{\mathrm{t}} \varphi_{1 B}^{\prime} \psi_{2 B}^{\prime}=0
$$

and it is found to be coincident with condition (32). It has the following interpretation: the virtual work done by viscous forces $\hat{\psi}$ in the displacements $\varphi$ is zero at $Z$ (but non-zero elsewhere along $\mathcal{D}$ ).

(b) Hopf boundary

If $\lambda=\mathrm{i} \omega$, then $p$ and $q$ are real (Equation (26)). By substituting them in matrix $\mathbf{R}$ and separating the real and imaginary parts of $\operatorname{det}(\mathbf{R})$, two real equations are obtained (see the Appendix), namely $\mathcal{H}_{i}(\mu, \kappa, \omega)=0(i=1,2)$. They define a codimension- 1 curve $\mathcal{H}$ in the bifurcation parameter plane, shown in Figure 3 for different $\xi_{\mathrm{t}}$ and $\alpha=0.5$. According to the Takens-Bogdanova bifurcation 


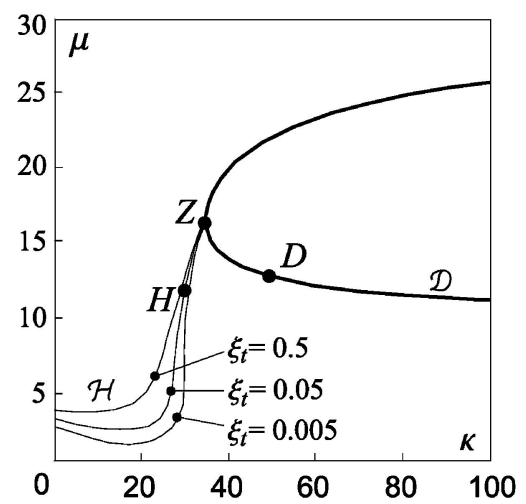

Figure 3. Linear stability diagram $\left(\mathcal{D}\right.$ : divergence boundary, $\mathcal{H}$ : Hopf boundary); $\alpha=\xi_{\mathrm{e}} / \xi_{\mathrm{t}}=0.5$.

mechanism (see [10]), the Hopf boundary dies when it collides with the divergence boundary at the double-zero point $Z$. The right and left eigenvectors at a selected point $H \equiv(30.082,11.932)$ on a $\mathcal{H}$-curve are reported in the appendix.

\section{Bifurcation Analysis}

The Multiple-Scale Method [17] is applied to analyze the system's behavior around a divergence point (say $D$, in Figure 3), a Hopf point (say $H$ ) and the double-zero point $(Z$ ). A perturbation parameter $\varepsilon$ is introduced as a measure of the distance of a given point from the bifurcation point in the parameter space. Different $\varepsilon$-dependent time scales $t_{k}=\varepsilon^{k} t$ are defined and the state variables $\mathbf{U}$ are expanded in Taylor series of $\varepsilon$. By equating terms of the same power of $\varepsilon$, linear perturbation equations having the same operator are obtained and then solved in sequence for the series coefficients $\mathbf{U}_{k}(k=1,2, \ldots)$. Except for the lower-order eigenvalue problem, higher-order equations are non-homogeneous of the type:

$$
\begin{gathered}
\left(\mathbf{B} \frac{d}{d t}-\mathbf{A}\right) \mathbf{U}_{k}=\mathbf{F}_{k} e^{\lambda t_{0}} \\
\mathbf{G} \mathbf{U}_{k}=\mathbf{0} \quad k=2,3, \ldots
\end{gathered}
$$

with $t_{0}$ being the fast time scale and $\lambda$ the critical eigenvalue. By letting $\mathbf{U}_{k}\left(s, t_{0}\right)=\mathbf{W}_{k}(s) e^{\lambda t_{0}}$, the problems:

$$
\begin{aligned}
& (\mathbf{A}-\lambda \mathbf{B}) \mathbf{W}_{k}=-\mathbf{F}_{k} \\
& \mathbf{G W}_{k}=\mathbf{0} \quad k=2,3, \ldots
\end{aligned}
$$

must be solved, with $\mathbf{A}-\lambda \mathbf{B}$ singular. Equations (35) admit a solution if and only if $\mathbf{F}_{k}$ belongs to the range of the singular operator, i.e. if:

$$
\left(\boldsymbol{\Psi}, \mathbf{F}_{k}\right)=0 \quad \forall \Psi:\left(\mathbf{A}^{*}-\bar{\lambda} \mathbf{B}^{*}\right) \Psi=\mathbf{0}
$$

Solvability equations (36), when combined on the true time scale $t$ (according to the so-called reconstitution method [18]), furnish the bifurcation equations, governing the asymptotic dynamics of the 
system, reduced to the center manifold. The procedure is explained in detail in the following, first for codimension-1 and then for codimension-2 bifurcations.

\subsection{CODIMENSION-1 BIFURCATIONS}

A single parameter, e.g. the load $\mu$, describes the transition through the bifurcation point. Let us denote by:

$$
\gamma \equiv \varepsilon^{2} \hat{\gamma}:=\mu-\mu_{0}
$$

the deviation of $\mu$ with respect to the bifurcation value, with $\varepsilon \ll 1$ and $O(\hat{\gamma})=1$. Accordingly:

$$
\mathbf{K}=\mathbf{K}_{0}+\varepsilon^{2} \hat{\gamma} \mathbf{K}_{\gamma}+O\left(\varepsilon^{4}\right)
$$

where $\mathbf{K}_{0}$ is the stiffness at the critical point and $\mathbf{K}_{\gamma}$ its derivative with respect to $\gamma$ at the same point. The state variables $\mathbf{U}$ are then expanded in series of $\varepsilon$ and independent time scales $t_{k}$ introduced. By accounting for the symmetry of the system, only odd powers are retained and only even time scales are considered, namely:

$$
\mathbf{U}=\varepsilon\left\{\begin{array}{c}
\mathbf{u} \\
\hat{\mathbf{u}}_{1}
\end{array}\right\}+\varepsilon^{3}\left\{\begin{array}{c}
\mathbf{u}_{3} \\
\hat{\mathbf{u}}_{3}
\end{array}\right\}+\cdots, \quad d / d t=d_{0}+\varepsilon^{2} d_{2}+\cdots
$$

with $d_{k}=\partial / \partial t_{k}$ and $t_{k}=\varepsilon^{k} t(k=0,2, \ldots)$.

From the first-order form (12) of the equations of motion, the following perturbation equations, up to $\varepsilon^{3}$-order, are derived:

$$
\begin{array}{ll} 
& d_{0} \mathbf{u}_{1}-\hat{\mathbf{u}}_{1}=\mathbf{0} \\
\varepsilon: \quad \mathbf{M} d_{0} \hat{\mathbf{u}}_{1}+\mathbf{K}_{0} \mathbf{u}_{1}+\mathbf{C} \hat{\mathbf{u}}_{1}=\mathbf{0} \\
& u_{1 A}=0, \quad u_{1 A}^{\prime}=0 \\
& \\
\varepsilon^{3}: \quad & d_{0} \mathbf{u}_{3}-\hat{\mathbf{u}}_{3}=-d_{2} \mathbf{u}_{1} \\
& \mathbf{M} d_{0} \hat{\mathbf{u}}_{3}+\mathbf{K}_{0} \mathbf{u}_{3}+\mathbf{C} \hat{\mathbf{u}}_{3}=-\mathbf{M} d_{2} \hat{\mathbf{u}}_{1}-\gamma \mathbf{K}_{\gamma} \mathbf{u}_{1}+\mathbf{n}\left(\mathbf{U}_{1}^{3}\right) \\
& u_{3 A}=0, \quad u_{3 A}^{\prime}=0
\end{array}
$$

where the hat has been omitted on $\gamma$. The static and dynamic cases are studied separately.

(a) Divergence

When $\lambda=0$, the first-order equations (40) furnish:

$$
\left\{\begin{array}{c}
\mathbf{u}_{1} \\
\hat{\mathbf{u}}_{1}
\end{array}\right\}=a\left(t_{2}, t_{4}, \ldots\right)\left\{\begin{array}{c}
\varphi \\
\mathbf{0}
\end{array}\right\}
$$

where $a=a\left(t_{2}, t_{4}, \ldots\right)$ is a real arbitrary constant. By using Equation (42), the third-order equation (41) reads:

$$
\begin{aligned}
& d_{0} \mathbf{u}_{3}-\hat{\mathbf{u}}_{3}=-d_{2} a \varphi \\
& \mathbf{M} \delta_{0} \hat{\mathbf{u}}_{3}+\mathbf{K}_{0} \mathbf{u}_{3}+\mathbf{C} \hat{\mathbf{u}}_{3}=-\gamma a \mathbf{K}_{\gamma} \boldsymbol{\varphi}+a^{3} \mathbf{n}\left(\Phi^{3}\right) \\
& u_{3 A}=0, \quad u_{3 A}^{\prime}=0
\end{aligned}
$$


By enforcing solvability (36), with $\mathbf{F}_{3}=\left\{-d_{2} a \varphi,-\gamma a \mathbf{K}_{\gamma} \boldsymbol{\varphi}+a^{3} \mathbf{n}\left(\Phi^{3}\right)\right\}^{\mathrm{T}}$, and $\boldsymbol{\Psi}=$ $\left\{0, \xi_{\mathrm{e}} \psi_{B}, \xi_{\mathrm{t}} \psi_{B}^{\prime} ; \psi, \psi_{B}, \psi_{B}^{\prime}\right\}^{\mathrm{T}}$ and reabsorbing the perturbation parameter, it follows:

$$
\dot{a}+c_{1 \gamma} a \gamma+c_{3} a^{3}=0
$$

where $c_{1 \gamma}$ and $c_{3}$ are real coefficients given in the appendix. Equation (44) is the bifurcation equation for the divergence.

(b) Hopf bifurcation

When $\lambda=i \omega$, Equations (40) furnish:

$\left\{\begin{array}{c}\mathbf{u}_{1} \\ \hat{\mathbf{u}}_{1}\end{array}\right\}=A\left(t_{2}, t_{4}, \ldots\right)\left\{\begin{array}{c}\varphi \\ \mathrm{i} \omega \varphi\end{array}\right\} e^{\mathrm{i} \omega t_{0}}+$ c.c.

where $A=A\left(t_{2}, t_{4}, \ldots\right)$ is a complex arbitrary constant and c.c. denotes the complex conjugate. By substituting Equation (45), Equation (41) becomes:

$$
\begin{aligned}
& d_{0} \mathbf{u}_{3}-\hat{\mathbf{u}}_{3}=-d_{2} A \varphi e^{\mathrm{i} \omega t_{0}}+\text { c.c. } \\
& \mathbf{M} d_{0} \hat{\mathbf{u}}_{3}+\mathbf{K}_{0} \mathbf{u}_{3}+\mathbf{C} \hat{\mathbf{u}}_{3}=\left(-\mathrm{i} \omega d_{2} A \mathbf{M} \boldsymbol{\varphi}-\gamma A \mathbf{K}_{\gamma} \varphi+3 A^{2} \bar{A} \mathbf{n}\left(\Phi^{2} \overline{\boldsymbol{\Phi}}\right)\right) e^{\mathrm{i} \omega t_{0}}+\mathrm{NST}+\text { c.c. } \\
& u_{3 A}=0, \quad u_{3 A}^{\prime}=0
\end{aligned}
$$

where NST stands for non-secular terms. Again, by accounting for $\mathbf{F}_{3}=\left\{-d_{2} A \varphi,-\mathrm{i} \boldsymbol{\omega} d_{2} A \mathbf{M} \boldsymbol{\varphi}-\right.$ $\left.\gamma A \mathbf{K}_{\gamma} \boldsymbol{\varphi}+3 A^{2} \bar{A} \mathbf{n}\left(\boldsymbol{\Phi}^{2} \overline{\boldsymbol{\Phi}}\right)\right\}^{\mathrm{T}}$ and $\boldsymbol{\Psi}=\left\{\bar{\lambda} \psi, \xi_{\mathrm{e}} \psi_{B}, \xi_{\mathrm{t}} \psi_{B}^{\prime} ; \psi, \psi_{B}, \psi_{B}^{\prime}\right\}^{\mathrm{T}}$ the solvability condition (36), after reabsorbing $\varepsilon$, leads to:

$$
\dot{A}+C_{1 \gamma} A \gamma+C_{3} A^{2} \bar{A}=0
$$

where $C_{1 \gamma}$ and $C_{3}$ are complex coefficients given in the appendix. Equation (47) is the bifurcation equation for the Hopf bifurcation.

\subsection{CODIMENSION-2 BIFURCATION}

The double-zero bifurcation requires the introduction of two bifurcation parameters. By taking the load $\mu$ and the stiffness $\kappa$ as bifurcation parameters, their increments with respect to the critical values $\mu_{0}$ and $\kappa_{0}$ are denoted by:

$$
\gamma \equiv \varepsilon^{2} \hat{\gamma}:=\mu-\mu_{0}, \quad \beta \equiv \varepsilon^{2} \hat{\beta}:=\kappa-\kappa_{0}
$$

with $O(\hat{\gamma})=O(\hat{\beta})=1$. Accordingly:

$$
\mathbf{K}=\mathbf{K}_{0}+\varepsilon^{2}\left(\hat{\beta} \mathbf{K}_{\beta}+\hat{\gamma} \mathbf{K}_{\gamma}\right)+O\left(\varepsilon^{4}\right)
$$

Expansion of the variables is less straightforward. Since the linear operator is defective (i.e. the set of eigenvectors is not complete at the bifurcation), fractional power expansions should be employed according to the treatment of the algebraic systems $[11,12]$. Since the eigenvalue multiplicity is equal to 2 , the appropriate expansion for a generic system contains powers of $\varepsilon^{1 / 2}$. However, due to the reflection 
symmetry of the system under study, only even powers of $\varepsilon^{1 / 2}$ are meaningful, so that a standard series expansion of $\varepsilon$ is recovered. By adopting for the time scales the same $\varepsilon$ dependence, it follows:

$$
\mathbf{U}=\sum_{k=1,2, \ldots} \varepsilon^{k}\left\{\begin{array}{l}
\mathbf{u}_{k} \\
\hat{\mathbf{u}}_{k}
\end{array}\right\}, \quad d / d t=\sum_{k=0,1, \ldots} \varepsilon^{k} d_{k}
$$

with $d_{k}=\partial / \partial t_{k}$ and $t_{k}=\varepsilon^{k} t(k=0,1,2, \ldots)$. Equations (48) and (50) lead to the following perturbation equations, up to the $\varepsilon^{4}$ order:

$$
\begin{array}{ll} 
& d_{0} \mathbf{u}_{1}-\hat{\mathbf{u}}_{1}=\mathbf{0} \\
\varepsilon: & \mathbf{M} d_{0} \hat{\mathbf{u}}_{1}+\mathbf{K}_{0} \mathbf{u}_{1}+\mathbf{C} \hat{\mathbf{u}}_{1}=\mathbf{0} \\
& u_{1 A}=0, \quad u_{1 A}^{\prime}=0 \\
& d_{0} \mathbf{u}_{2}-\hat{\mathbf{u}}_{2}=-d_{1} \mathbf{u}_{1} \\
\varepsilon^{2}: \quad \mathbf{M} d_{0} \hat{\mathbf{u}}_{2}+\mathbf{K}_{0} \mathbf{u}_{2}+\mathbf{C} \hat{\mathbf{u}}_{2}=-\mathbf{M} d_{1} \hat{\mathbf{u}}_{1} & u_{2 A}=0, \quad u_{2 A}^{\prime}=0 \\
& d_{0} \mathbf{u}_{3}-\hat{\mathbf{u}}_{3}=-d_{2} \mathbf{u}_{1}-d_{1} \mathbf{u}_{2} \\
\varepsilon^{3}: \quad \mathbf{M} d_{0} \hat{\mathbf{u}}_{3}+\mathbf{K}_{0} \mathbf{u}_{3}+\mathbf{C} \hat{\mathbf{u}}_{3}=-\mathbf{M} d_{2} \hat{\mathbf{u}}_{1}-\mathbf{M} d_{1} \hat{\mathbf{u}}_{2}-\beta \mathbf{K}_{\beta} \mathbf{u}_{1}-\gamma \mathbf{K}_{\gamma} \mathbf{u}_{1}+\mathbf{n}\left(\mathbf{U}_{1}^{3}\right) \\
& u_{3 A}=0, \quad u_{3 A}^{\prime}=0 \\
& d_{0} \mathbf{u}_{4}-\hat{\mathbf{u}}_{4}=-d_{3} \mathbf{u}_{1}-d_{2} \mathbf{u}_{2}-d_{1} \mathbf{u}_{3} \\
& \mathbf{M} d_{0} \hat{\mathbf{u}}_{4}+\mathbf{K}_{0} \mathbf{u}_{4}+\mathbf{C} \hat{\mathbf{u}}_{4}=-\mathbf{M} d_{3} \hat{\mathbf{u}}_{1}-\mathbf{M} d_{2} \hat{\mathbf{u}}_{2}-\mathbf{M} d_{1} \hat{\mathbf{u}}_{3} \\
\varepsilon^{4}: \quad-\beta \mathbf{K}_{\beta} \mathbf{u}_{2}-\gamma \mathbf{K}_{\gamma} \mathbf{u}_{2}+3 \mathbf{n}\left(\mathbf{U}_{1}^{2} \mathbf{U}_{2}\right) \\
\\
u_{4 A}=0, \quad u_{4 A}^{\prime}=0 \quad
\end{array}
$$

where the hat has been omitted on $\beta$ and $\gamma$. As a normalization condition, $u_{B}=1$ is adopted, entailing $u_{1 B}=1$ and $u_{k B}=0$ for $k>1$.

The eigenvalue problem (51) admits the (generating) solution:

$$
\left\{\begin{array}{l}
\mathbf{u}_{1} \\
\hat{\mathbf{u}}_{1}
\end{array}\right\}=a\left(t_{1}, t_{2}, \ldots\right)\left\{\begin{array}{c}
\varphi_{1} \\
\mathbf{0}
\end{array}\right\}
$$

where $a\left(t_{1}, t_{2}, \ldots\right)$ is a real, arbitrary amplitude. With the previous equation, the $\varepsilon^{2}$-order problem reads:

$$
\begin{aligned}
& d_{0} \mathbf{u}_{2}-\hat{\mathbf{u}}_{2}=-d_{1} a \boldsymbol{\varphi}_{1} \\
& \mathbf{M} d_{0} \hat{\mathbf{u}}_{2}+\mathbf{K}_{0} \mathbf{u}_{2}+\mathbf{C} \hat{\mathbf{u}}_{2}=\mathbf{0} \\
& u_{2 A}=0, \quad u_{2 A}^{\prime}=0
\end{aligned}
$$

Since the known term belongs to the range of the operator (recall Equations (24)), Equations (56) admit the steady (not diverging) solution:

$$
\left\{\begin{array}{c}
\mathbf{u}_{2} \\
\hat{\mathbf{u}}_{2}
\end{array}\right\}=d_{1} a\left(t_{1}, t_{2}, \ldots\right)\left\{\begin{array}{c}
\varphi_{2} \\
\varphi_{1}
\end{array}\right\}
$$

in which $\boldsymbol{\Phi}_{2}=\left\{\varphi_{2} \hat{\varphi}_{2}\right\}^{\mathrm{T}}=\left\{\varphi_{2} \varphi_{1}\right\}^{\mathrm{T}}$ is the order-2 generalized eigenvector associated with $\lambda=0$. It should be noted that $d_{1} a$ is still undetermined at this order. With the previous results, the $\varepsilon^{3}$-order 
equations become:

$$
\begin{aligned}
& d_{0} \mathbf{u}_{3}-\hat{\mathbf{u}}_{3}=-d_{2} a \boldsymbol{\varphi}_{1}-d_{1}^{2} a \boldsymbol{\varphi}_{2} \\
& \mathbf{M} d_{0} \hat{\mathbf{u}}_{3}+\mathbf{K}_{0} \mathbf{u}_{3}+\mathbf{C} \hat{\mathbf{u}}_{3}=-d_{1}^{2} a \mathbf{M} \boldsymbol{\varphi}_{1}-\beta a \mathbf{K}_{\beta} \boldsymbol{\varphi}_{1}-\gamma a \mathbf{K}_{\gamma} \boldsymbol{\varphi}_{1}+a^{3} \mathbf{n}\left(\Phi_{1}^{3}\right) \\
& u_{3 A}=0, \quad u_{3 A}^{\prime}=0
\end{aligned}
$$

In order to solve these equations, the known term $\mathbf{F}_{3}=\left\{-d_{2} a \varphi_{1}-d_{1}^{2} a \varphi_{2},-d_{1}^{2} a \mathbf{M} \varphi_{1}-\right.$ $\left.\beta a \mathbf{K}_{\beta} \boldsymbol{\varphi}_{1}-\gamma a \mathbf{K}_{\gamma} \boldsymbol{\varphi}_{1}+a^{3} \mathbf{n}\left(\boldsymbol{\Phi}_{1}^{3}\right)\right\}^{\mathrm{T}}$ must be made orthogonal to the proper left eigenvector $\boldsymbol{\Psi}_{2}=$ $\left\{\bar{\lambda} \psi_{2}, \xi_{\mathrm{e}} \psi_{2 B}, \xi_{t} \psi_{2 B}^{\prime} ; \psi_{2}, \psi_{2 B}, \psi_{2 B}^{\prime}\right\}^{\mathrm{T}}$, by requiring $\left(\boldsymbol{\Psi}_{2}, \mathbf{F}_{3}\right)=0$; this entails that:

$$
d_{1}^{2} a=\left(c_{1 \beta} \beta+c_{1 \gamma} \gamma\right) a+c_{3} a^{3}
$$

where $c_{1 \beta}, c_{1 \gamma}$ and $c_{3}$ are real coefficients. By using Equation (59), and solving Equation (58), it follows:

$$
\mathbf{U}_{3}=\left\{\begin{array}{c}
\mathbf{u}_{3} \\
\hat{\mathbf{u}}_{3}
\end{array}\right\}=d_{2} a\left\{\begin{array}{c}
\boldsymbol{\varphi}_{2} \\
\boldsymbol{\varphi}_{1}
\end{array}\right\}+\beta a\left\{\begin{array}{c}
\mathbf{z}_{\beta} \\
\hat{\mathbf{z}}_{\beta}
\end{array}\right\}+\gamma a\left\{\begin{array}{c}
\mathbf{z}_{\gamma} \\
\hat{\mathbf{z}}_{\gamma}
\end{array}\right\}+a^{3}\left\{\begin{array}{c}
\mathbf{z}_{a} \\
\hat{\mathbf{z}}_{a}
\end{array}\right\}
$$

Equation (60) is found as (unique) steady solution of Equations (58) and the normalization condition $u_{3 B}=0$; this is reported in the appendix. By using the results achieved, the $\varepsilon^{4}$-order perturbation equation reads:

$$
\begin{aligned}
& d_{0} \mathbf{u}_{4}-\hat{\mathbf{u}}_{4}=-d_{3} a \varphi_{1}-d_{1} d_{2} a \varphi_{2}-d_{1} \mathbf{u}_{3} \\
& \mathbf{M} d_{0} \hat{\mathbf{u}}_{4}+\mathbf{K}_{0} \mathbf{u}_{4}+\mathbf{C} \hat{\mathbf{u}}_{4}=-d_{1} d_{2} a \mathbf{M} \varphi_{1}-\mathbf{M} d_{1} \hat{\mathbf{u}}_{3} \\
& \quad-\beta d_{1} a \mathbf{K}_{\beta} \varphi_{2}-\gamma d_{1} a \mathbf{K}_{\gamma} \varphi_{2}+3 a^{2} d_{1} a \mathbf{n}\left(\Phi_{1}^{2} \Phi_{2}\right) \\
& u_{4 A}=0, \quad u_{4 A}^{\prime}=0
\end{aligned}
$$

The solvability of these equations entails:

$$
2 d_{1} d_{2} a=\left(e_{1 \beta} \beta+e_{1 \gamma} \gamma\right) d_{1} a+e_{3} a^{2} d_{1} a
$$

where the real coefficients $e_{1 \beta}, e_{1 \gamma}$ and $e_{3}$ are defined in appendix. By returning to the true time, Equations (59) and (62) are recombined, furnishing:

$$
\ddot{a}=\left(c_{1 \beta}+c_{1 \gamma}\right) a+\left(e_{1 \beta}+e_{1 \gamma}\right) \dot{a}+c_{3} a^{3}+e_{3} a^{2} \dot{a}
$$

to within an error of order $\varepsilon^{5}$. Equation (63) is the bifurcation equation for the double-zero bifurcation; it directly appears in the Bogdanova-Arnold normal form.

\section{Numerical Results for the Double-Zero Bifurcation}

By introducing the coefficients $\chi:=-\left(c_{1 \beta} \beta+c_{1 \gamma} \gamma\right)$ and $\delta:=-\left(e_{1 \beta} \beta+e_{1 \gamma} \gamma\right)$ (unfolding parameters), Equation (63) reads:

$$
\ddot{a}+\delta \dot{a}+\chi a-c_{3} a^{3}-e_{3} a^{2} \dot{a}=0
$$




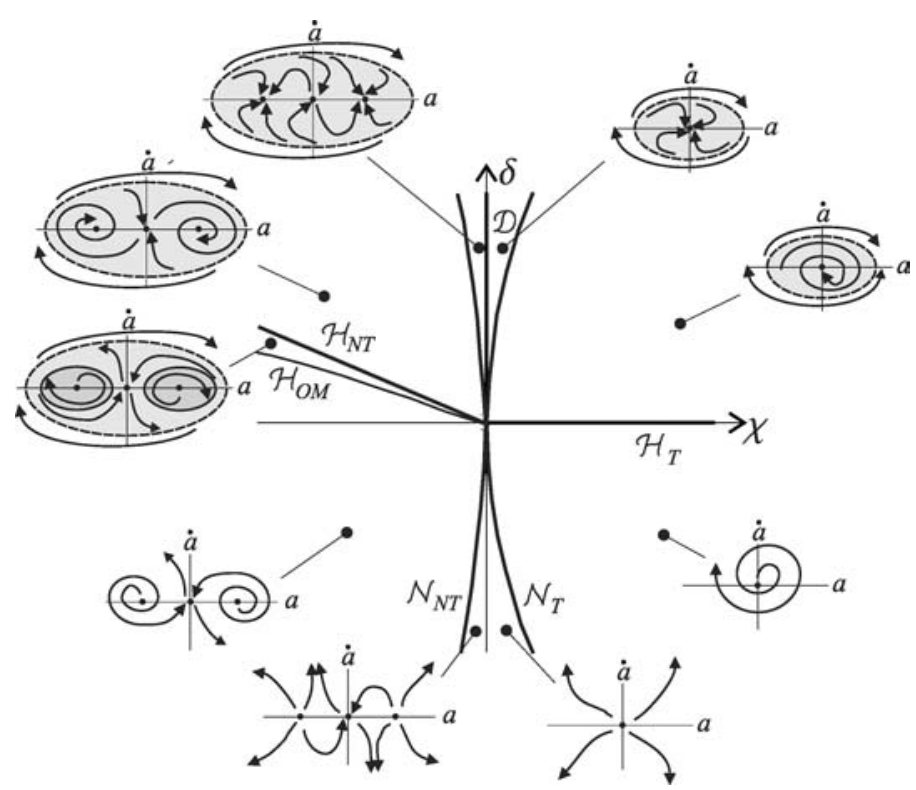

Figure 4. Bifurcation diagram around the double-zero point: unfolding parameter plane and phase-plane sketches.

A qualitative analysis of Equation (64) leads to the bifurcation diagram of Figure 4. The $\delta$-axis is a divergence boundary, while the positive $\chi$-axis is a Hopf boundary for the trivial solution (here denoted by $\mathcal{H}_{\mathrm{T}}$ ). Along the Hopf boundary an under-critical bifurcation occurs and an unstable limit cycle arises (large cycles in Figure 4, in which the system oscillates around the trivial solution). The cycle exists in the angular sector bounded by the $\mathcal{H}_{\mathrm{T}}$ and $\mathcal{H}_{\mathrm{OM}}$ straight lines. Along the divergence boundary a pitchfork bifurcation takes place, for which two non-trivial equilibria emerge, describing symmetrical buckled configurations of the beam. The non-trivial equilibria exist in the half-plane $\chi<0$; they are stable above the straight line $\mathcal{H}_{\mathrm{NT}}$ and unstable below this line. At $\mathcal{H}_{\mathrm{NT}}$ the non-trivial solution undergoes a Hopf bifurcation from which two stable limit cycles arise (small cycles in Figure 4, in which the system oscillates around a buckled configuration). However, they only exist in a small region of the plane bounded by the straight lines $\mathcal{H}_{\mathrm{NT}}$ and $\mathcal{H}_{\mathrm{OM}}$. Indeed, at $\mathcal{H}_{\mathrm{OM}}$, a homoclinic bifurcation takes place, caused by the multiple collision between the (small) limit cycle, the saddle point at the origin, and the large cycle. In conclusion, stable solutions exist only in the region above the straight lines $\mathcal{H}_{\mathrm{T}}$ and $\mathcal{H}_{\mathrm{OM}}$ of the plane: the trivial equilibrium for $\chi>0, \delta>0$; two stable buckled equilibria between $\mathcal{H}_{\mathrm{NT}}$ and $\mathcal{D}$; the small cycles between $\mathcal{H}_{\mathrm{NT}}$ and $\mathcal{H}_{\mathrm{OM}}$. In addition to the previously defined boundaries, two further curves, $\mathcal{N}_{\mathrm{T}}$ and $\mathcal{N}_{\mathrm{NT}}$, organize the bifurcation diagram, which are loci of (nilpotent) systems possessing two coincident eigenvalues.

\section{Conclusions}

The efficiency of the Multiple-Scale Method in obtaining reduced-order models of infinite-dimensional systems has been shown. The method applies to continuous systems in a way formally equal to that of discrete systems, widely discussed in earlier works. The method consists in the following steps: 
(1) The linear differential operator, which includes the mechanical boundary conditions, accounting for lumped viscoelastic devices and/or mass, is considered. After having defined a scalar product, the adjoint operator and the adjoint boundary conditions are derived from the bilinear identity.

(2) The spectrum of the linear operator is analyzed to find linear stability boundaries of the trivial equilibrium position in the parameter space. Both right and left eigenvectors are evaluated at the critical points. If the operator is defective, i.e. if its eigenvectors do not form a complete set (this occurs for non-self-adjoint operators when an eigenvalue has multiplicity larger than 1), then generalized eigenvectors must be evaluated (said to form a Keldysh chain), similarly to the eigenvectors of an algebraic operator (said to form a Jordan chain).

(3) The state variables are then expanded in series of a perturbation parameter $\varepsilon$; the bifurcation parameters are scaled so that they first appear at the first meaningful order; several independent time scales are introduced; the linear perturbation equations are derived by collecting terms of the same $\varepsilon$ order.

(4) The perturbation equations are solved in sequence, which calls for the right-hand side to be orthogonal to the left eigenvectors. These solvability conditions supply amplitude equations on different time scales.

(5) By recombining the amplitude equations and returning to the true time scale, the bifurcation equations capturing the asymptotic dynamics of the system are recovered. They are found to be already in normal form.

The procedure has been illustrated for a continuous model of a planar beam, internally constrained, equipped with a viscoelastic device and loaded by a follower force. The system exhibits three instability forms: (a) divergence, (b) Hopf bifurcation and (c) double-zero bifurcation, all analyzed in the paper. Due to the simplicity of the model, it was possible to find (by Mathematica ${ }^{\circledR}$ ) closed-form equations for the eigenvectors and the coefficients of the bifurcation equations. It is expected that a numerical approach will be necessary for more involved equations.

The paper represents an initial attempt to address the problem. More complex systems should be analyzed (e.g. exhibiting quadratic nonlinearities, responsible for modification of the bifurcation pattern of the structure) and/or more complex interactions studied (e.g. among resonant critical eigenvalues). However, the strict analogy with algebraic problems holds hope that the method can also be applied in these cases.

\section{Appendix}

Right and left (proper and generalized) eigenvectors at points $D, H$ and $Z$ of Figure 3 are evaluated. The analytical expressions for the coefficients of the relevant bifurcation equations are then reported, together with the numerical values they assume at the selected points, useful in future numerical investigations.

\section{(a) Divergence}

When $\lambda=0$, Equation (25) reads:

$$
\varphi(s)=c_{1}(\cos p s-1)+c_{2}(\sin p s-p s)
$$




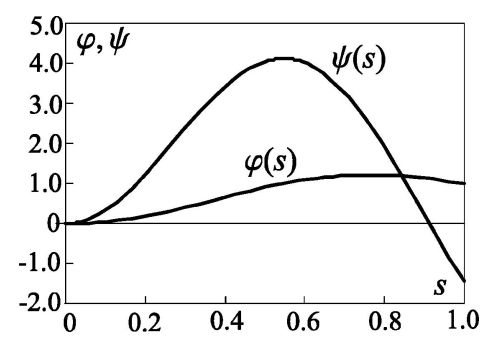

Figure 5. Right $\varphi(s)$ and left $\psi(s)$ eigenvectors of the divergence point $D$ of Figure 3.

with $p=\sqrt{2 \mu}$. By letting $\alpha=0.5, \xi_{t}=0.05$, assuming $\kappa=50, \mu=12.688$ (point $D$ in Figure 3) and solving Equation (27), one has:

$$
\boldsymbol{\Phi}:=\left\{\begin{array}{c}
\varphi \\
\varphi_{B} \\
\varphi_{B}^{\prime} \\
\lambda \varphi \\
\lambda \varphi_{B} \\
\lambda \varphi_{B}^{\prime}
\end{array}\right\}=c_{1}\left\{\begin{array}{c}
-1-1.70 s+\cos (5.04 s)+0.34 \sin (5.04 s) \\
-2.70 \\
3.62 \\
0 \\
0 \\
0
\end{array}\right\}
$$

By requiring $\varphi_{B}=1, c_{1}=-0.370$ follows; the right eigenvector is plotted in Figure 5 .

The adjoint problem (22) admits the solution:

$$
\psi(s)=d_{1}(\cos p s-1)+d_{2}(\sin p s-p s)
$$

which satisfies only geometrical conditions. By enforcing the mechanical conditions and solving them, one finds:

$$
\boldsymbol{\Psi}:=\left\{\begin{array}{c}
\bar{\lambda} \psi \\
\xi_{\mathrm{e}} \psi_{B} \\
\xi_{\mathrm{t}} \psi_{B}^{\prime} \\
\psi \\
\psi_{B} \\
\psi_{B}^{\prime}
\end{array}\right\}=d_{1}\left\{\begin{array}{c}
0 \\
0.013 \\
0.27 \\
-1-s+\cos (5.04 s)-0.20 \sin (5.04 s) \\
0.51 \\
5.45
\end{array}\right\}
$$

with $d_{1}$ undetermined. By normalizing according to $(\boldsymbol{\Psi}, \boldsymbol{\Phi})=1, d_{1}=-2.833$ is drawn; the left eigenvector is plotted in Figure 5.

The coefficients appearing in the bifurcation equation (44) are:

$$
c_{1 \gamma}=\int_{0}^{1} 2 \varphi^{\prime \prime} \psi d s, \quad c_{3}=-\left[n_{2}(\boldsymbol{\Phi})^{3} \psi_{B}+n_{3}(\boldsymbol{\Phi})^{3} \psi_{B}^{\prime}+\int_{0}^{1} n_{1}\left(\boldsymbol{\Phi}^{3}\right) \psi d s\right]
$$

where $n_{i}(\cdot)(i=1,2,3)$ are the components of $\mathbf{n}(\cdot)$ given by Equation (116). Coefficients (69), evaluated at $D$, assume the following values: $c_{1 \gamma}=-18.146$ and $c_{3}=723.573$.

(b) Simple Hopf 

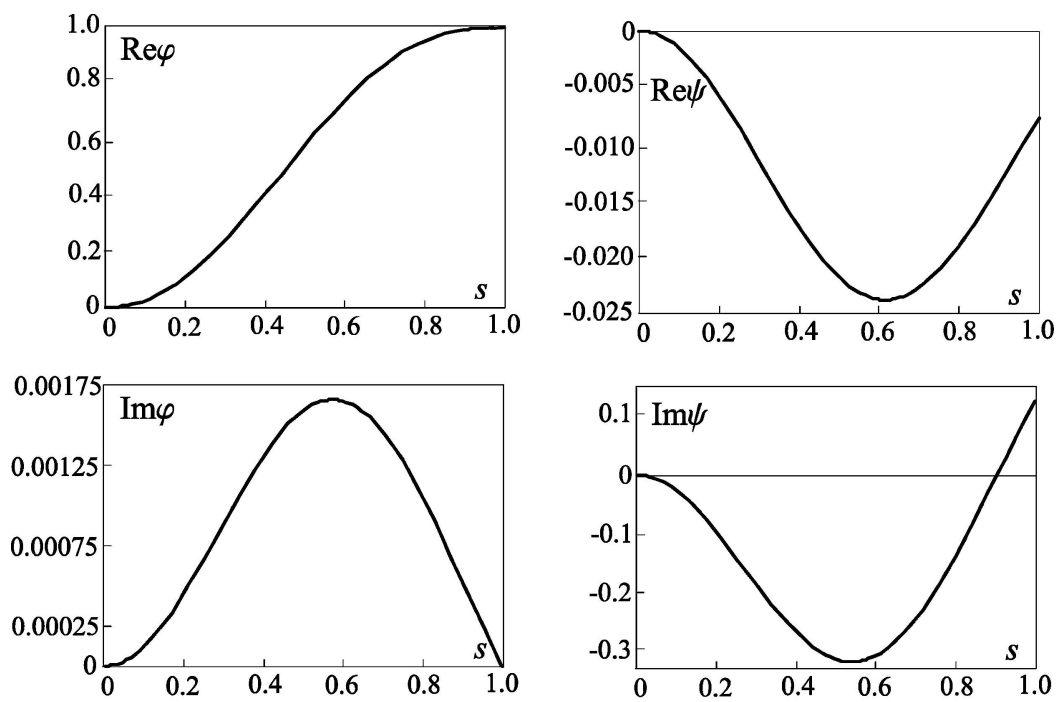

(a)

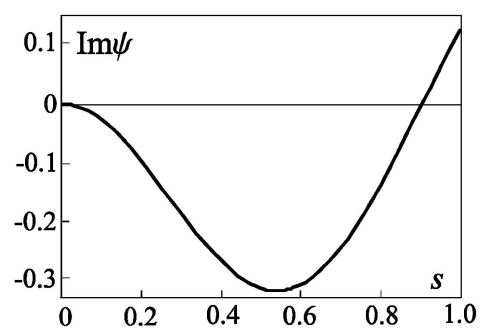

(b)

Figure 6. Right $\varphi(s)$ and left $\psi(s)$ eigenvectors of the Hopf point $H$ of Figure 3.

When $\lambda=\mathrm{i} \omega$, Equation (25) holds, with $q^{2}=\sqrt{\mu^{2}+\omega^{2}}-\mu, p^{2}=\sqrt{\mu^{2}+\omega^{2}}+\mu$. By expanding the determinant of the matrix $\mathbf{R}$ in the Equation (27), the following two equations are found, defining the Hopf boundary $\mathcal{H}$ :

$$
\begin{aligned}
& p q\left(p^{4}+q^{4}-2 \alpha \xi_{\mathrm{t}}^{2} \omega^{2}\right)+q \cosh q\left[2 p\left(p^{2} q^{2}+\alpha \xi_{\mathrm{t}}^{2} \omega^{2}\right) \cos p+\left(p^{2}+q^{2}\right) \kappa \sin p\right] \\
& \quad-\left[p\left(p^{2}+q^{2}\right) \kappa \cos p+\left(p^{2}+q^{2}\right)\left(p^{2} q^{2}+\alpha \xi_{\mathrm{t}}^{2} \omega^{2}\right) \sin p\right] \sinh q=0 \\
& 2 p q \kappa+q \cosh q\left[-2 p \kappa \cos p+\left(p^{2}+q^{2}\right)\left(p^{2}+\alpha\right) \sin p\right] \\
& \quad+\left[p\left(p^{2}+q^{2}\right)\left(q^{2}-\alpha\right) \cos p+\left(q^{2}-p^{2}\right) \kappa \sin p\right] \sinh q=0
\end{aligned}
$$

By fixing $\alpha=0.5$ and $\xi_{\mathrm{t}}=0.05$, a point $H$ (Figure 3) of coordinates $\kappa=30.082, \mu=11.932$ is found on $\mathcal{H}$, at which $\omega=5.2$. By solving Equation (27), the relevant right eigenvector is found:

$$
\Phi:=\left\{\begin{array}{c}
\varphi \\
\varphi_{B} \\
\varphi_{B}^{\prime} \\
\lambda \varphi \\
\lambda \varphi_{B} \\
\lambda \varphi_{B}^{\prime}
\end{array}\right\}=c_{1}\left\{\begin{array}{c}
\cos (4.99 s)-\cosh (1.04 s)+(0.50-0.004 \mathrm{i})(\sin (4.99 s)-4.80 \sinh (1.04 s)) \\
-4.74+0.026 \mathrm{i} \\
0.25+0.025 \mathrm{i} \\
5.2 \mathrm{i}[\cos (4.99 s)-\cosh (1.04 s)+(0.50-0.004 \mathrm{i})(\sin (4.9 s)-4.80 \sinh (1.04 s))] \\
-0.13-24.64 \mathrm{i} \\
-0.13+1.31 \mathrm{i}
\end{array}\right\}
$$

in which $c_{1}=-(0.211+0.00115 \mathrm{i})$ follows from $\varphi_{B}=1$. The real and imaginary parts of $\varphi$ are illustrated in Figure 6a.

The adjoint problem (22) admits the solution:

$$
\psi(s)=d_{1}(\cos p s-\cosh q s)+d_{2}\left(\sin p s-\frac{p}{q} \sinh q s\right)
$$


from which the left eigenvector is drawn:

$$
\Psi:=\left\{\begin{array}{c}
\bar{\lambda} \psi \\
\xi_{\mathrm{e}} \psi_{B} \\
\xi_{\mathrm{t}} \psi_{B}^{\prime} \\
\psi \\
\psi_{B} \\
\psi_{B}^{\prime}
\end{array}\right\}=d_{1}\left\{\begin{array}{c}
-5.2 \mathrm{i}[\cos (4.99 s)-\cosh (1.04 s)+(0.27+0.009 \mathrm{i})(\sin (4.99 s)-4.80 \sinh (1.04 s))] \\
0.014+0.0016 \mathrm{i} \\
0.26+0.0031 \mathrm{i} \\
\cos (4.99 s)-\cosh (1.04 s)+(0.27+0.009 \mathrm{i})(\sin (4.99 s)-4.80 \sinh (1.04 s)) \\
0.56+0.064 \mathrm{i} \\
5.29+0.061 \mathrm{i}
\end{array}\right\}
$$

After normalization $(\boldsymbol{\Psi}, \boldsymbol{\Phi})=1, d_{1}=-(0.0523+0.178 \mathrm{i})$ follows. Eigenvector $\psi$ is shown in Figure 6b.

The coefficients of the bifurcation equation (47) assume the form:

$$
C_{1 \gamma}=\int_{0}^{1} 2 \varphi^{\prime \prime} \psi d s, \quad C_{3}=-\left[n_{2}\left(\boldsymbol{\Phi}^{2} \overline{\boldsymbol{\Phi}}\right) \psi_{B}+n_{3}\left(\boldsymbol{\Phi}^{2} \overline{\boldsymbol{\Phi}}\right) \psi_{B}^{\prime}+\int_{0}^{1} n_{1}\left(\boldsymbol{\Phi}^{2} \overline{\boldsymbol{\Phi}}\right) \psi d s\right]
$$

Coefficients (74), evaluated at $H$, assume the following values: $C_{1 \gamma}=-(0.0140+0.479 \mathrm{i})$ and $C_{3}=(0.958+38.327 \mathrm{i})$.

(c) Double zero

Equation (65) also holds at the double-zero point $Z$. By fixing $\alpha=0.5, Z$ occurs at $\kappa=34.820$ and $\mu=16.470$ (Figure 3). For $\xi_{\mathrm{t}}=0.05$ the right eigenvector is found to be:

$$
\Phi_{1}:=\left\{\begin{array}{c}
\varphi_{1} \\
\varphi_{1 B} \\
\varphi_{1 B}^{\prime} \\
\lambda \varphi_{1} \\
\lambda \varphi_{1 B} \\
\lambda \varphi_{1 B}^{\prime}
\end{array}\right\}=c_{1}\left\{\begin{array}{c}
-1-9.51 s+\cos (5.074 s)+1.66 \sin (5.07 s) \\
-10.51 \\
1.60 \\
0 \\
0 \\
0
\end{array}\right\}
$$

By substituting it in Equations (24), solving them and accounting for geometrical conditions, it follows:

$$
\varphi_{2}(s)=a_{1}(\cos p s-1)+a_{2}(\sin p s-p s)
$$

where $a_{1}, a_{2}$ are arbitrary constants. Mechanical conditions call for solving Equation (31), where

$$
\mathbf{R}_{0}=\left[\begin{array}{cc}
-p^{2} \cos p & -p^{2} \sin p \\
\kappa(1-\cos p)+p^{3} \sin p & -p^{3} \cos p+\kappa(p-\sin p)
\end{array}\right], \quad \mathbf{f}=c_{1}\left\{\begin{array}{c}
-p \xi_{\mathrm{t}} \tan (p / 2) \\
\xi_{\mathrm{e}}(1-p \cot p)
\end{array}\right\}
$$

Matrix $\mathbf{R}_{0}$ is singular at $Z$, but $\mathbf{f}$ belongs to its range; therefore Equation (31) can be solved for $\left\{a_{1}, a_{2}\right\}$ to within one constant. The generalized eigenvector therefore reads:

$$
\boldsymbol{\Phi}_{2}:=\left\{\begin{array}{c}
\varphi_{2} \\
\varphi_{2 B} \\
\varphi_{2 B}^{\prime} \\
\varphi_{1} \\
\varphi_{1 B} \\
\varphi_{1 B}^{\prime}
\end{array}\right\}=\left\{\begin{array}{c}
a_{1}[-1-9.51 s+\cos (5.74 s)+1.66 \sin (5.74 s)] \\
+c_{1}[0.027 s-0.0047 \sin (5.74 s)] \\
0.029 c_{1}-10.51 a_{1} \\
0.0039 c_{1}+1.60 a_{1} \\
a_{1}[-1-9.51 s+\cos (5.074 s)+1.66 \sin (5.07 s)] \\
-10.51 a_{1} \\
1.60 a_{1}
\end{array}\right\}
$$




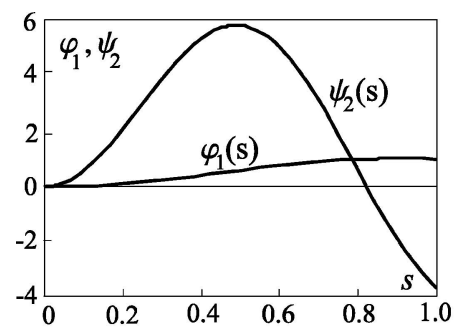

(a)

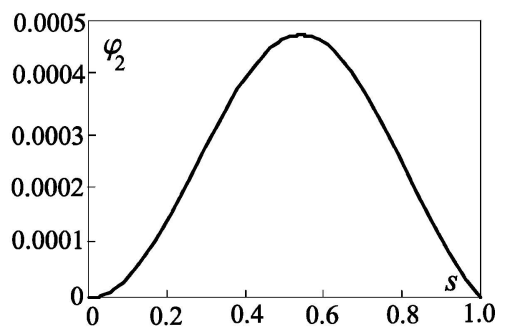

(b)

Figure 7. Proper $\left(\varphi_{1}(s), \psi_{2}(s)\right)$ and generalized $\left(\varphi_{2}(s)\right)$ eigenvectors at the double-zero point $Z$ of Figure 3 .

The arbitrary constants $c_{1}$ and $a_{1}$ are determined respectively by imposing $\varphi_{1 B}=1$ and $\varphi_{2 B}=0$; then $c_{1}=-0.0952, a_{1}=-0.000266$. The right eigenvectors $\varphi_{1}$ and $\varphi_{2}$ are shown in Figure $7 \mathrm{a}$ and $\mathrm{b}$.

The adjoint problem admits the field solution (67), which satisfies the geometrical conditions. From the mechanical conditions, it follows:

$$
\Psi_{2}:=\left\{\begin{array}{c}
\bar{\lambda} \psi_{2} \\
\xi_{\mathrm{e}} \psi_{2 B} \\
\xi_{\mathrm{t}} \psi_{2 B}^{\prime} \\
\psi_{2} \\
\psi_{2 B} \\
\psi_{2 B}^{\prime}
\end{array}\right\}=d_{1}\left\{\begin{array}{c}
0 \\
0.024 \\
0.16 \\
-1+s+\cos (5.74 s)-0.17 \sin (5.74 s) \\
0.95 \\
3.11
\end{array}\right\}
$$

By requiring $\left(\boldsymbol{\Psi}_{2}, \boldsymbol{\Phi}_{2}\right)=1, d_{1}=-3.718$ is found. The left eigenvector $\psi_{2}$ is plotted in Figure 7 a.

To build up the bifurcation equation (63), the $\mathbf{z}$-solutions appearing in Equation (60) must first be evaluated. They satisfy the following problems:

$$
\begin{aligned}
& d_{0} \mathbf{z}_{\beta}-\hat{\mathbf{z}}_{\beta}=-c_{1 \beta} \boldsymbol{\varphi}_{2} \\
& \mathbf{M} d_{0} \hat{\mathbf{z}}_{\beta}+\mathbf{K}_{0} \mathbf{z}_{\beta}+\mathbf{C} \hat{\mathbf{z}}_{\beta}=-c_{1 \beta} \mathbf{M} \boldsymbol{\varphi}_{1}-\mathbf{K}_{\beta} \boldsymbol{\varphi}_{1} \\
& z_{\beta A}=0, \quad z_{\beta A}^{\prime}=0 \\
& d_{0} \mathbf{z}_{\gamma}-\hat{\mathbf{z}}_{\gamma}=-c_{1 \gamma} \boldsymbol{\varphi}_{2} \\
& \mathbf{M} d_{0} \hat{\mathbf{z}}_{\gamma}+\mathbf{K}_{0} \mathbf{z}_{\gamma}+\mathbf{C} \hat{\mathbf{z}}_{\gamma}=-c_{1 \gamma} \mathbf{M} \boldsymbol{\varphi}_{1}-\mathbf{K}_{\gamma} \boldsymbol{\varphi}_{1} \\
& z_{\gamma A}=0, \quad z_{\gamma A}^{\prime}=0 \\
& d_{0} \mathbf{z}_{a}-\hat{\mathbf{z}}_{a}=-c_{3} \boldsymbol{\varphi}_{2} \\
& \mathbf{M} d_{0} \hat{\mathbf{z}}_{a}+\mathbf{K}_{0} \mathbf{z}_{a}+\mathbf{C} \hat{\mathbf{z}}_{a}=-c_{3} \mathbf{M} \boldsymbol{\varphi}_{1}-\mathbf{n}\left(\Phi_{1}^{3}\right) \\
& z_{a A}=0, \quad z_{a A}^{\prime}=0
\end{aligned}
$$

under the normalization conditions:

$$
z_{\beta B}=0, \quad z_{\gamma B}=0, \quad z_{a B}=0
$$

and assume cumbersome expressions, not reported here.

Coefficients in Equation (63) take the following forms:

$$
\begin{aligned}
c_{1 \beta} & =-\varphi_{1 B} \psi_{2 B}, \quad c_{1 \gamma}=2\left(\varphi_{1 B}^{\prime} \psi_{2 B}-\varphi_{1 B} \psi_{2 B}^{\prime}\right) \\
c_{3} & =-\int_{0}^{1} \psi_{2} n_{1}\left(\boldsymbol{\Phi}_{1}^{3}\right) d s+\psi_{2 B} n_{2}\left(\boldsymbol{\Phi}_{1}^{3}\right)+\psi_{2 B}^{\prime} n_{3}\left(\boldsymbol{\Phi}_{1}^{3}\right)
\end{aligned}
$$


and

$$
\begin{aligned}
e_{1 \beta} & =-\left(\int_{0}^{1} \psi_{2} \hat{z}_{\beta} d s+\xi_{\mathrm{e}} \psi_{2 B} z_{\beta B}+\xi_{\mathrm{t}} \psi_{2 B}^{\prime} z_{\beta B}^{\prime}+\psi_{2 B} \varphi_{2 B}\right) \\
e_{1 \gamma} & =-\left(\int_{0}^{1} \psi_{2} \hat{z}_{\gamma} d s+\xi_{\mathrm{e}} \psi_{2 B} z_{\gamma B}+\xi_{\mathrm{t}} \psi_{2 B}^{\prime} z_{\gamma B}^{\prime}-2 \psi_{2 B} \varphi_{2 B}^{\prime}+2 \psi_{2 B}^{\prime} \varphi_{2 B}\right) \\
e_{3} & =-\left[\int_{0}^{1} \psi_{2}\left(\hat{z}_{a}-n_{1}\left(\boldsymbol{\Phi}_{1}^{2} \boldsymbol{\Phi}_{2}\right)\right) d s+\xi_{\mathrm{e}} \psi_{2 B} z_{a B}+\xi_{\mathrm{t}} \psi_{2 B}^{\prime} z_{a B}^{\prime}-\psi_{2 B} n_{2}\left(\boldsymbol{\Phi}_{1}^{2} \boldsymbol{\Phi}_{2}\right)-\psi_{2 B}^{\prime} n_{3}\left(\boldsymbol{\Phi}_{1}^{2} \boldsymbol{\Phi}_{2}\right)\right]
\end{aligned}
$$

Coefficients (84) and (85), evaluated at $Z$, assume the following values: $c_{1 \beta}=-3.517, c_{1 \gamma}=0.503$, $e_{1 \beta}=4.303, e_{1 \gamma}=-7.107, c_{3}=-137.960$ and $e_{3}=231.472$.

\section{Acknowledgements}

This research was supported by the Italian Ministry for Universities and Scientific Research MIUR (Cofin 2003-04 funds).

\section{References}

1. Steindl, A. and Troger, H., 'Methods for dimension reduction and their application in nonlinear dynamics', International Journal of Solids and Structures 38, 2001, 3131-2147.

2. Nayfeh, A. H., 'Reduced-order models of weakly nonlinear spatially continuous systems', Nonlinear Dynamics 16, 1998, 105-125.

3. Nayfeh, A. H. and Lacarbonara, W., 'On the discretization of spatially continuous systems with quadratic and cubic nonlinearities', JSME International Journal 41, 1998, 510-531.

4. Rega, G., Lacarbonara, W., Nayfeh, A. H., and Chin, C., 'Multiple resonances in suspended cables: Direct versus reducedorder models', International Journal of Non-Linear Mechanics 34, 1999, 901-924.

5. Rega, G., Lacarbonara, W., and Nayfeh, A. H., 'Reduction methods for nonlinear vibrations of spatially continuous systems with initial curvature', Solid Mechanics and Its Applications 77, 2000, 235-246.

6. Nayfeh, A. H., Arafat, H., Chin, C. M., and Lacarbonara, W., 'Multimode interactions in suspended cables', Journal of Vibration and Control 8, 2002, 337-387.

7. Luongo, A. and Paolone, A., 'Perturbation methods for bifurcation analysis from multiple nonresonant complex eigenvalues', Nonlinear Dynamics 14, 1997, 193-210.

8. Luongo, A. and Paolone, A., 'Multiple scale analysis for divergence-Hopf bifurcation of imperfect symmetric systems', Journal Sound and Vibration 218, 1998, 527-539.

9. Luongo, A., Paolone, A., and Di Egidio, A., 'Multiple time scales analysis for 1:2 and 1:3 resonant Hopf bifurcations', Nonlinear Dynamics 34(3-4), 2003, 269-281.

10. Luongo, A., Paolone, A., and Di Egidio, A., 'Sensitivity and linear stability analysis around a double zero eigenvalues', AIAA Journal 38(4), 2000, 702-710.

11. Luongo, A., Di Egidio, A., and Paolone, A., 'Multiple time scale analysis for bifurcation from a multiple-zero eigenvalue', AIAA Journal 41(6), 2003, 1143-1150.

12. Luongo, A., Di Egidio, A., and Paolone, A., 'Multiple scale bifurcation analysis for finite-dimensional autonomous systems', in Recent Research Developments in Sound \& Vibration, Transworld Research Network, Kerala, India, Vol. 1, 2002, pp. 161-201.

13. Nayfeh, A. H., Problems in Perturbation, Wiley, New York, 1985.

14. Kirillov, O. N. and Seyranian, A. P., 'Collapse of Keldysh chain and stability of nonconservative systems', Doklady Mathematics 66(1), 2002, 127-131 (Translated from Doklady Mathematics Nauk 385(2), 2002, 172-176).

15. Kirillov, O. N. and Seyranian, A. P., 'Solution to the Herrmann-Smith problem', Doklady Physics 47(10), 2002, 767-771 (from Doklady Akademii Nauk 386(6), 2002, 761-766).

16. Keldysh, M. V., 'On eigenvalues and eigenfunctions of certain classes of not self-adjoint equations', Doklady Akademii Nauk SSRR 77(1), 1951, 11-14.

17. Nayfeh, A. H., Introduction to Perturbation Techniques, Wiley-Interscience, New York, 1981.

18. Nayfeh, A. H., 'Topical Course on Nonlinear Dynamics', Società Italiana di Fisica, Santa Margherita di Pula, Sardinia, Perturbation Methods in Nonlinear Dynamics, 1985. 